\title{
Heterogeneous Models and Modelling Approaches for Engineering of Interactive Systems
}

The selection of articles that follow were solicited from attendees of several workshops that are related in their focus on engineering methods for interactive systems design and development. The first of these was the Workshop on Heterogeneous Models and Modelling Approaches for Engineering of Interactive Systems, which took place in Paris in June 2018, colocated with the 10th ACM SIGCHI Symposium on Engineering Interactive Computing Systems (EICS). This workshop focussed on the development, investigation and use of heterogeneous models and modelling methods in the context of user interfaces, interaction techniques, and interactive systems. Presentations at this workshop were based on themes such as heterogeneous models and heterogeneous modelling approaches; formal, semi-formal or informal models and languages; perspectives on the creation, semi-automatic or automatic generation of software parts of interactive systems using a heterogeneous modelling approach and model transformations.

Additionally, invitations to submit were extended to attendees of the $7^{\text {th }}$ and $8^{\text {th }}$ Formal Methods for Interactive Systems Workshops that took place in Toulouse, in June 2018, as an affiliated workshop of STAF 2018 and in Porto, in October 2019, co-located with Formal Methods 2019. The FMIS workshops aim to bring together researchers who are interested in both formal methods and interactive system design. More specifically, in the development and application of formal methods, as well as in related verification techniques and analysis tools, in the context of Human-Computer Interaction $(\mathrm{HCl})$. An additional focus is on the general design and verification methodologies, which take account of models or as well as the complexity of human behaviour (both at development time (developers) and operation time (users)).

Attendees of these workshops together with interested researchers in related fields were invited to submit papers for this collection based on the topics and themes from the workshops above. Nine papers were submitted and six of these were accepted following revisions. The accepted papers address a range of topics, including hyper-connected cars, medical devices, flight training procedures, and aircraft cockpits. They describe the use of a variety of formalisms such as Petri nets, task models, Event-B, PVS, etc., and demonstrate the breadth of engineering techniques and tools that can be applied to multi-modal interactive systems from diverse application domains.

Most of the contributions are built on existing research contributions in the area of formal methods and software engineering. They extend, refine or combine these contributions to address the complex nature of interactive systems and user interfaces. Some of them address the engineering of these interactive systems; others address the verification of individual or combined properties; while others focus on the improvement of their use and usability.

In the domain of Human-Computer Interaction where a vast amount of resources are invested in the User Experience property, the papers in this special issue address other important properties such as usability, reliability, and correctness with respect to requirements. Therefore, the papers in this special issue address multiple safety critical domains 
(automotive, healthcare and avionics) where correctness with respect to requirements is critical. Some of them also address the under-studied issue (in the field of $\mathrm{HCl}$ ) of certification of devices or systems by trusted third parties. Another difference with mainstream $\mathrm{HCl}$ is that most of the papers in this special issue propose engineering solutions to address correctness of WIMP interaction techniques, which is not fashionable anymore in that domain even though unsolved issues with their design and engineering remain. There are two papers, however, targeting at more complex interaction techniques involving Augmented Reality or smartwatches devices but still in safety critical domains (automotive and aircraft cockpits).

We believe these research contributions are valuable and complementary to the classical contributions both in software engineering and $\mathrm{HCl}$.

Toulouse, December $18^{\text {th }} 2020$

Yamine Aït-Ameur, Judy Bowen, José Creissac Campos, Philippe Palanque \& Benjamin Weyers Organizers of the special issue. 